Pensamiento Crítico Vol. 21, № 2, pp. 105 - 126

\title{
Percepción de los niños sobre la violencia $e$ inseguridad urbana y la probabilidad de ser víctimas. Caso Lima Metropolitana y Callao
}

\author{
Perception of children on violence and urban insecurity and the probability \\ of being victims. Lima Metropolitan and Callao case.
}

[Recepción: Setiembre de 2016 / Conformidad: Octubre de 2016]

Juan León Mendoza ${ }^{1}$

\section{RESUMEN}

El artículo tiene como objetivo fundamental conocer y cuantificar el grado de victimización y la percepción sobre la violencia e inseguridad urbana de los niños de Lima Metropolitana y Callao. Para ello se aplicó una encuesta a 364 niños de 8-12 años de edad.

Según los resultados de la encuesta, los niños perciben al robo y al asalto en la calle como el problema más importante. La tasa de victimización en los niños, relacionado a los robos y asaltos en la calle, es $14 \%$. Los niños son víctimas de

1 Docente principal e investigador asociado al Instituto de Investigaciones Económicas de la Facultad de Ciencias Económicas de la Universidad Nacional Mayor de San Marcos.

Este artículo es una versión resumida de la investigación "Percepción de los niños sobre la violencia e inseguridad urbana en Lima Metropolitana y Callao", efectuada en el marco del Concurso Multidisciplinario de Investigación 2015 del VRI-UNMSM. Participaron también en la investigación: Miguel Inga Arias, Pablo Rivas Santos, Andrés Zavala Abel, Richard Roca Garay, Julio Alvarado Vera, Greis Nahuinmallma Aliaga, María Elena Abarca Gonzáles. 


\section{Pensamiento Crítico Vol. ᄅl, № 2}

violencia física en mayor grado en el hogar (36\%) que en el colegio $(22,3 \%)$ y la calle $(12,6 \%)$.

El 77\% de los encuestados se sienten inseguros o poco seguros en las calles fuera de su barrio. Su grado de confianza en las instituciones encargadas de velar por la seguridad urbana es baja: un $60 \%$ confía poco o no confía en la policía. En tanto que para el caso del serenazgo es $78 \%$.

Los niños opinan que el principal factor por el que las personas se dedican al robo y asalto está en que no les gusta trabajar y "se la quieren llevar fácil".

La regresión econométrica del modelo logit indica que la probabilidad de que un niño sufra algún robo o asalto en la calle es mayor en la medida en que este pertenezca a un hogar disfuncional y tenga poca o ninguna confianza en sus padres.

Palabras clave: Inseguridad urbana, violencia urbana, violencia niños, robos y asaltos.

\section{ABSTRACT}

This article aims essentially to understand and quantify the degree of victimization and the perception of violence and urban insecurity of children in Metropolitan Lima and Callao. In order to do this we took a survey to 364 children aged between 8-12 years old.

According to survey results, children perceive theft and assault in the street as the most important problem. The rate of victimization in children related to thefts and robberies on the than in school $(22,3 \%)$ or streets $(12,6 \%)$.

$77 \%$ of respondents feel insecure or unsafe on the streets outside their neighborhood. Their confidence in the institutions responsible for urban safety is low: About $60 \%$ have little or no trust in the police, and even worse in watchmen (78\%).

Children believe that the main factor of why people engage in robbery and assault is that they do not like to work and they want easy carrying.

Our logit econometric regression model indicates that the probability of children suffering some theft or assault in the street is higher as long as they belong to a dysfunctional home and have little or no trust in their parents.

Keywords: Urban insecurity, urban violence, children violence, robberies and assaults. 


\section{Juan León Mendoza}

\section{Introducción}

Según la encuesta de la Municipalidad de Lima Metropolitana, Lima Cómo Vamos (2014), aplicada a personas mayores de 15 años, el problema más importante que afecta a los ciudadanos de Lima Metropolitana es la delincuencia y la inseguridad urbana (82\%). Según la citada encuesta, el 49,6\% de los encuestados fueron víctimas de robo en la calle, en el domicilio, en un local de transporte público, o agredidos $\mathrm{u}$ hostigados en la calle.

Las encuestas y los estudios al respecto usualmente recogen la percepción de las personas mayores de edad. No existen estudios a nivel de los niños.

Por ello, se torna muy relevante efectuar una investigación sobre el grado de percepción que tienen los niños sobre la creciente violencia e inseguridad urbana que se observa en Lima Metropolitana y Callao, en la medida que dicho fenómeno afecta su desarrollo socioemocional y tiende a formar una generación de personas propensas a la violencia, que luego obstaculizarán el avance económico y el crecimiento económico (Hofstetter, 1998).

\section{Marco teórico y antecedentes}

En principio, el concepto de violencia es muy complejo, difuso y se modifica en el tiempo y el espacio en la medida en que los valores y las normas sociales cambian. Según la Organización Panamericana de la Salud (2002, p.6), la violencia se define como "el uso deliberado de la fuerza física o el poder, ya sea en grado de amenaza o efectivo, contra uno mismo, otra persona o un grupo o comunidad, que cause o tenga muchas probabilidades de causar lesiones, muerte, daños psicológicos, trastornos del desarrollo o privaciones". En tal sentido, la violencia puede ser de naturaleza física, sexual, psicológica y de privaciones o desatenciones. A su vez se clasifican en autoinflingida, interpersonal y colectiva. La violencia autoinflingida puede ser de comportamiento suicida y autolesiones. La violencia interpersonal se subclasifica en violencia intrafamiliar y la comunitaria. La violencia a nivel de la familia puede generarse contra los menores, la pareja o los ancianos. La violencia colectiva puede ser de carácter social, político y económico. 


\section{Pensamiento Crítico Vol. ᄅl, № 2}

En este marco conceptual, se entiende por violencia urbana a aquellas acciones o actividades que generan daños físicos, sexuales, psicológicos, y privaciones que ocurren en las áreas urbanas o metrópolis.

Si bien la violencia urbana se localiza en las ciudades o urbes, esta se tiende a describir en función de cinco variables: características directas e indirectas, intensidad y duración, características espaciales y sociales, intencionalidad y contexto. También se suele aproximar la definición de violencia urbana a los actos criminales, cuyas manifestaciones más importantes son: robos a mano armada, agresiones, homicidios o secuestros (Vanderschueren, 1994). También, según Del Olmo (2000), la violencia urbana se asocia a actos criminales que generan lesiones o daños físicos a las personas y que, por ende, también se manifiestan en la forma de atracos, robos, tentativas de homicidios, violación, violencia familiar, lesiones de tránsito terrestre, homicidios.

En el Perú no se registran estudios sobre la victimización y la percepción sobre la violencia urbana a nivel de niños, lo que sí existe son trabajos relacionados solo a la violencia intrafamiliar e institucional. Entre los trabajos de violencia intrafamiliar, contra los niños, podemos citar el caso de Ponce (1995). En tanto que, referido a la violencia institucional en las escuelas y en la forma de bullying, se puede citar lo efectuado por Oliveros et al. (2008).

En el escenario internacional, un estudio sobre la violencia de tipo cualitativo y de grupos focales de niños y adolescentes efectuado por la ONU (2006), para un grupo de 17 países latinoamericanos, arrojó resultados interesantes. Según dicho estudio, los niños manifiestan convivir cotidianamente con la violencia y perciben cuatro formas de violencia: la física, la psicológica, la sexual y la discriminación. Identifican discriminación por origen racial o étnico, origen socioeconómico, sexo o género, capacidades diferentes y rango etario. Mencionan a los padres, madres, padrastros y hermanos o hermanas mayores como los principales agresores en el hogar; en tanto que en la calle, a los hombres en general y a sus pares; a nivel institucional, a los policías, las enfermeras y los doctores; en la escuela, a los maestros o maestras y a sus pares. La violencia por género se da básicamente hacia las niñas y los adolescentes. Sostienen que la violencia es generada básicamente por la pobreza, la falta de acceso a la educación, el desempleo y el incumplimiento de los derechos básicos de alimentación o salud, que a su vez conducen al consumo de diversos tipos de droga. Reconocen que la violencia genera más violencia, lo cual afecta la imagen que tienen de sí mismos, además de ocasionarles 


\section{Juan León Mendoza}

perjuicios en su salud física. Declaran que, ante los problemas de violencia, acuden en son de protección a sus amistades más que a sus padres y familiares, en el marco de que estos últimos son los que generan la mayor violencia.

Relacionado a las consecuencias o efectos que generan la violencia en los niños, existen estudios a nivel internacional que ponen de manifiesto que estos son negativos y muy importantes.

Según Perry (1997), la violencia en la comunidad sobre los niños genera daños neurobiológicos profundos y duraderos. La violencia afecta negativamente su sentimiento de seguridad y confianza en sí mismo, llegando inclusive a perdurar en períodos posteriores a su niñez. En el mismo sentido, Sheidow et al. (2001) indican que los niños inmersos en una comunidad violenta presentan -durante su infancia y juventudcuadros o índices altos de estrés, depresión y trastornos mentales. Según Osofsky (1999), en comunidades con reiterados actos de violencia, los niños ven afectados negativamente su desarrollo social, de modo que tienden a presentar dificultades para establecer relaciones de confianza no solo durante su niñez, sino también en edades adultas. Los homicidios que se producen en la comunidad generan desarreglos de atención, afectando de manera grave la capacidad de aprendizaje y rendimiento de los niños (Sharkey, 2010). Los niños que viven en comunidades violentas tienden a ser más violentos y agresivos con otros niños (Dodge et al., 1990).

\section{Metodología}

Dado nuestro objetivo central de conocer y cuantificar la percepción de los niños sobre la violencia e inseguridad urbana, se aplicó una encuesta a 364 niños de 8-12 años de edad en los distritos de Lima Metropolitana y Callao. Dicho tamaño de muestra es estadísticamente representativo debido a que según el Instituto Nacional de Estadística e Informática (2015), el universo de la población total de niños comprendidos entre la citada edad es igual a 740,625.

Con la finalidad de efectuar el análisis por estratos socioeconómicos, tomando el criterio de los distritos y según el Índice de Desarrollo Humano (IDH) del PNUD, se determinaron los siguientes cuatro niveles socioeconómicos: 


\section{Pensamiento Crítico Vol. ᄅl, № 2}

A = Miraflores, La Molina, Lince, San Isidro, La Punta, Jesús María, San Borja, Pueblo Libre, San Miguel, Magdalena, Barranco, Surco. B = Surquillo, Breña, San Luis, La Perla, Bellavista, Lima, Santa María del Mar, Chaclacayo, Rímac, Los Olivos, Chorrillos. C = San Martín de Porres, La Victoria, San Juan de Miraflores, Santa Anita, Punta Hermosa, Comas, Independencia, San Bartolo, San Juan de Lurigancho, Santa Rosa, El Agustino, Callao, Chosica. D = Ate, Villa El Salvador, Ancón, Carmen de la Legua, Villa María del Triunfo, Lurín, Punta Negra, Puente Piedra, Carabayllo, Cieneguilla, Ventanilla, Pucusana.

Los distritos con el mayor IDH están agrupados en el nivel A, en tanto que el nivel D comprende a los de menor IDH.

Dado que el robo y asalto es el principal problema de violencia e inseguridad que aqueja a la capital peruana, estimaremos econométricamente los factores que determinan la probabilidad de que el niño sea víctima de ello. Dicha probabilidad se estimará utilizando el modelo logit.

\section{Nivel de percepción y victimización}

\section{Principales problemas sobre violencia e inseguridad}

Según los niños encuestados, el problema más importante, sobre violencia e inseguridad urbana en Lima Metropolitana y Callao, es el robo y el asalto en la calle (36,3\%). Luego, sigue el problema de consumo de drogas (12,1\%), el bullying (11\%), la violación $(10,4 \%)$ y los asesinatos (10,2\%), tal como se puede visualizar en el cuadro 1.

En relación con los robos y asaltos, las niñas, más que los niños, tienen la percepción de que este es el problema más importante. Si bien el pandillaje aparece como el sexto problema más importante según los niños encuestados, el $62,1 \%$ observó peleas de pandilleros en su zona de residencia. 


\section{Juan León Mendoza}

CUADRO 1

Percepción sobre el problema de violencia e inseguridad más importante

\begin{tabular}{|l|l|}
\hline Problemas & Porcentaje (\%) \\
\hline Robos o asaltos en la calle & 36,3 \\
\hline Consumo de drogas & 12,1 \\
\hline Bullying & 11,0 \\
\hline Violación & 10,4 \\
\hline Asesinatos & 10,2 \\
\hline Pandillaje & 9,3 \\
\hline Robo de vivienda & 6,0 \\
\hline Secuestros & 4,7 \\
\hline Fuente: Encuesta ejecutada & \\
\hline
\end{tabular}

\section{Nivel de victimización en robos y asaltos}

El 14\% de los encuestados fueron víctimas de algún robo o asalto en las calles de Lima y Callao y el $12,1 \%$ en su hogar. Por sexo, la tasa de victimización de los robos y asaltos en la calle es muy similar. Sin embargo, por estratos socioeconómicos, existe cierta tendencia: A mayor nivel socioeconómico, hay una mayor tasa de robos y asaltos. Así, en los distritos de sector $\mathrm{D}$, el porcentaje de niños víctimas de este tipo de delitos fue $16 \%$, en tanto que en B y A, las tasas fueron $20,3 \%$ y $22,6 \%$ respectivamente.

\section{Grado de victimización en violencia física}

La violencia física contra los niños, en la forma de golpes y castigos físicos, es mayor en el hogar que en el colegio y la calle. El porcentaje de niños que sufrieron algún tipo de agresión física en su hogar fue $36 \%$, en tanto que la tasa correspondiente al centro educativo donde estudia o en la calle fue $22,3 \%$ y $12,6 \%$ respectivamente (ver cuadro 2).

La tasa de violencia física contra los niños en el hogar aumenta en la medida en que es mayor el nivel socioeconómico distrital. En los sectores D y C (relativamente más pobres) la tasa de agresión física contra los niños es $32,1 \%$ y 35,7\% respectivamente, en tanto que en los sectores B y A, las tasas son $39 \%$ y $45,2 \%$. 


\section{Pensamiento Crítico Vol. ᄅl, № 2}

Si bien, por estratos socioeconómicos, no existe una tendencia sobre la magnitud de la tasa de violencia física en las calles, por sexo sí se tiene una clara diferencia: la tasa de agresión física es mayor contra los niños que las niñas. El 16,8\% de los niños encuestados manifestaron haber sido víctimas de algún tipo de agresión física en la calle, en tanto que la cifra a nivel de las niñas fue $8,1 \%$.

\section{CUADRO 2}

Violencia física contra los niños, según lugar de ocurrencia (\%)

\begin{tabular}{|l|c|c|c|}
\hline \multicolumn{1}{|c|}{ Lugar } & Sí & No & NS/NR \\
\hline En el hogar & 36,0 & 64,0 & - \\
\hline En el colegio o escuela & 22,3 & 77,2 & 0,5 \\
\hline En la calle & 12,6 & 87,4 & - \\
\hline
\end{tabular}

Fuente: Encuesta ejecutada

\section{Nivel de victimización de violencia sexual}

La tasa de victimización en la violencia sexual hacia los niños (consistente en acciones de besos, caricias o insinuaciones morbosas por parte de personas distintas a sus padres o hermanos) es $8,2 \%$. La cifra es ligeramente mayor en los niños que en las niñas

\section{Percepción de seguridad e inseguridad urbana}

Los niños encuestados se sienten básicamente muy seguros en su hogar $(76,1 \%)$ $y$ en sus colegios o escuelas $(63,7 \%)$. La seguridad absoluta percibida es menor en las calles de su barrio $(33,5 \%)$ y en otras calles fuera de su barrio $(12,4 \%)$. La inseguridad es mayor en las calles fuera de su barrio. En dichas calles el $88,4 \%$ de los encuestados se sienten inseguros o poco seguros, en tanto que en las calles de su barrio el porcentaje es $65,4 \%$. Por estratos socioeconómicos, el porcentaje de niños que se sienten muy seguros en su hogar aumenta en la medida que se tiene un menor nivel de pobreza distrital. Es decir, en los distritos más pobres, el porcentaje de niños que se sienten muy seguros es mayor que en los distritos de estratos socioeconómicos altos.

Respecto a la percepción de seguridad en el colegio o la escuela, esta muestra una tendencia opuesta a la hallada a nivel de la seguridad en el hogar. El porcentaje de niños 


\section{Juan León Mendoza}

que muestran sentirse muy seguros en su centro educativo es mayor en la medida en que sube el nivel socioeconómico.

Sobre la seguridad en las calles de su barrio, el porcentaje de niños que se sienten muy seguros tiende a ser mayor en los distritos de nivel socioeconómico alto.

En relación con la seguridad en las calles fuera del barrio en que vive el niño, el porcentaje de aquellos que se sienten poco seguros aumenta en la medida en que disminuye el nivel socioeconómico distrital. La poca seguridad y la inseguridad que sienten los niños en la calle refleja cierta concordancia con la percepción de una débil protección que brinda la policía. Si bien el $55,5 \%$ de los niños se sienten protegidos por la policía, existe también un porcentaje alto, $42 \%$, que no se siente protegido.

\section{Percepción sobre la evolución de la violencia e inseguridad urbana}

Según la encuesta, en los últimos doce meses, para el $67 \%$ el problema de la violencia e inseguridad en Lima y Callao tendió a incrementarse. Por sexo, la percepción sobre la evolución creciente de la magnitud de la violencia es muy similar. Por niveles socioeconómicos, la percepción negativa tiende a ser mayor entre los niños de los distritos relativamente más pobres.

Existe un pesimismo generalizado sobre las perspectivas de la violencia $e$ inseguridad en Lima y Callao. El 50,3\% de los encuestados cree que, en los siguientes años, el problema seguirá igual; incluso un $31 \%$ percibe que el problema será más

grave. El pesimismo sobre la futura persistencia de la violencia, en los niveles que se perciben actualmente, es mayor entre las niñas que entre los niños.

\section{Grado de confianza}

En concordancia con el sentimiento de una mayor seguridad en el hogar y el colegio, muestran también una mayor confianza en sus padres y en sus profesores (ver cuadro 3). El $81,9 \%$ y el $69 \%$ de los niños encuestados confían mucho en sus padres y sus profesores, respectivamente. 


\section{Pensamiento Crítico Vol. ᄅl, № 2}

Predomina la poca confianza en los policías $(45,9 \%)$, en los compañeros de colegio $(51.6 \%)$, en los serenazgos $(46,4 \%)$ y en los vecinos mayores de edad de su barrio (46,4\%). Las mayores tasas de desconfianza absoluta están representadas por los vecinos adultos y los serenazgos.

El porcentaje de niños que confían mucho en sus padres es mayor que en el caso de las niñas $(83,8 \%$ contra $79,8 \%)$. Por estratos socioeconómicos, la tasa de los que confían mucho es mayor en los sectores socioeconómicamente bajos que en los altos.

Las niñas confían en menor grado en la Policía que los niños. El 54,9\% de las niñas confía poco en la Policía, en tanto que en el caso de los niños la cifra es 37,7\%. La tasa de los que confían mucho es $44,5 \%$ en los niños y $34,1 \%$ en las niñas.

CUADRO 3

Grado de confianza de los niños (\%)

\begin{tabular}{|l|c|c|c|c|}
\hline & Mucho & Poco & No confía & NS/NR \\
\hline Padres & 81,9 & 16,2 & 1,4 & 0,5 \\
\hline Profesores & 69,0 & 26,1 & 4,1 & 0,8 \\
\hline Policías & 39,6 & 45,9 & 14,3 & 0,2 \\
\hline Compañeros de colegio & 34,6 & 51,6 & 13,5 & 0,3 \\
\hline Serenazgos & 31,6 & 46,4 & 20,9 & 1,1 \\
\hline Vecinos mayores de edad & 26,4 & 46,4 & 26,1 & 1,1 \\
\hline
\end{tabular}

Fuente: Encuesta ejecutada

La tasa de baja confianza entre compañeros del colegio es un poco mayor en las niñas que en los niños. Asimismo, las niñas muestran una menor confianza en el serenazgo. En cuanto al grado de confianza en los vecinos que son mayores de edad, predomina ligeramente la desconfianza en las niñas que en los niños.

En resumen, las niñas, más que los niños, tienden a mostrar un menor grado de confianza en sus padres, sus compañeros de escuela, la Policía, el serenazgo y sus vecinos mayores de edad. 


\section{Juan León Mendoza}

\section{Razones de la violencia e inseguridad urbana: caso de los robos y los asaltos}

Según la opinión de los niños encuestados, entre las principales razones por las que las personas se dedican a los robos y asaltos, se sindica la propensión a la pereza $\mathrm{u}$ ociosidad $(35,2 \%)$, la falta de una buena educación en casa $(21,2 \%)$, la carencia de una buena niñez $(15,4 \%)$ y la necesidad económica $(13,5 \%)$. Ver cuadro 4.

\section{CUADRO 4}

Principal razón por la que algunas personas se dedican a los robos y los asaltos en Lima y Callao

\begin{tabular}{|l|c|}
\hline \multicolumn{1}{|c|}{ Razones } & Porcentaje (\%) \\
\hline Porque no les gusta trabajar y "se la quieren llevar fácil" & 35,2 \\
\hline Por falta de una buena educación en casa & 21,2 \\
\hline Porque no tuvieron buena niñez & 15,4 \\
\hline Por necesidad/ no tienen plata & 13,5 \\
\hline Porque no tienen valores & 9,3 \\
\hline Porque no estudian & 4,9 \\
\hline Consumo de drogas & 0,3 \\
\hline No van a la iglesia & 0,3 \\
\hline Fuente: Encuesta ejecutada & \\
\hline
\end{tabular}

\section{Propuestas de forma de castigo y solución}

El 79,9\% de los encuestados manifiestan que les preocupa mucho el problema de la violencia e inseguridad. La tasa de preocupación es mayor entre las niñas (85\%) que entre los niños $(75,4 \%)$.

En el marco de una gran preocupación, los niños sugieren que la principal forma de castigar a aquellos que delinquen consistiría en meterlos a la cárcel por un tiempo hasta que puedan reformarse $(45,2 \%)$ o aplicarles la cadena perpetua (35,6\%). El 10,4\% de los encuestados son partidarios de aplicarles la pena de muerte. 


\section{Pensamiento Crítico Vol. ᄅl, № 2}

Entre las principales propuestas que sugieren los niños para resolver el problema de los robos y la delincuencia urbana en general, destacan el incrementar la dotación de policías $(40,4 \%)$, elevar la eficiencia de la Policía (17\%), aplicar un castigo más fuerte $(15,9 \%)$ y poner más cámaras de vigilancia en zonas estratégicas (15,4\%). Ver cuadro 5.

\section{CUADRO 5}

Medidas para resolver el problema de la delincuencia urbana

\begin{tabular}{|l|c|}
\hline \multicolumn{1}{|c|}{ Medidas de solución } & Porcentaje (\%) \\
\hline Aumentar la seguridad con más policías & 40,4 \\
\hline Que la Policía sea más rápida para capturar a los delincuentes & 17,0 \\
\hline Aplicar un castigo más fuerte a los delincuentes & 15,9 \\
\hline Poner más cámaras de vigilancia & 15,4 \\
\hline Sacar el ejército a la calle & 8,2 \\
\hline Hacer deporte & 2,7 \\
\hline Implementar proyectos de rehabilitación en la Iglesia & 0,3 \\
\hline Fuente: Encuesta ejecutada & \\
\hline
\end{tabular}

\section{Probabilidad de ser víctima de un robo o asalto en la calle}

Utilizando la información recabada a través de la encuesta aplicada, a continuación efectuaremos un ejercicio de regresión econométrica con el modelo logit. Determinaremos los factores que explican la probabilidad de que el niño sea víctima de sufrir un robo o asalto en la calle.

El modelo funcional: $\quad Y=f(X 1, X 2, X 3, X 4)$

El modelo general: $\quad P\left(Y=1 / X^{\prime} \beta\right)=\frac{1}{1+e^{-X i \beta}}$ 


\section{Juan León Mendoza}

Variables:

Y: En el último año lo asaltaron; no $=0$, sí $=1$.

$\mathrm{X} 1$ : Con quien vive (mamá y papá=1, $2=$ con mamá o papá, 3 = otros)

$\mathrm{X} 2$ : Confianza en los padres (poco o nada $=0$, mucho $=1$ )

X3: Sexo (niña $=0$, niño $=1$ )

$\mathrm{X} 4$ : Distrito de residencia/ nivel socioeconómico $(\mathrm{A}=1, \mathrm{~B}=2, \mathrm{C}=3, \mathrm{D}=4)$.

CUADRO 6

Probabilidad de sufrir un robo o asalto en la calle

Dependent Variable: Y

Method: ML - Binary Logit (Quadratic hill climbing)

Date: 12/06/15 Time: 10:26

Sample: 1364

Included observations: 364

Convergence achieved after 5 iterations

Covariance matrix computed using second derivatives

\begin{tabular}{ccccc}
\hline \multicolumn{1}{c}{ Variable } & Coefficient & Std. Error & z-Statistic & Prob. \\
\hline C & -1.448995 & 0.690760 & -2.097684 & 0.0359 \\
X1 & 0.449101 & 0.228975 & 1.961351 & 0.0498 \\
X2 & -1.030077 & 0.339695 & -3.032358 & 0.0024 \\
X3 & 0.105682 & 0.311426 & 0.339350 & 0.7343 \\
X4 & -0.105002 & 0.171705 & -0.611527 & 0.5409 \\
\hline McFadden R-squared & 0.048939 & Mean dependentvar & 0.140110 \\
S.D. dependentvar & 0.347579 & S.E. of regression & 0.340151 \\
Akaikeinfocriterion & 0.798141 & Sum squaredresid & 41.53718 \\
Schwarzcriterion & 0.851674 & Log likelihood & -140.2617 \\
Hannan-Quinncriter. & 0.819418 & Deviance & 280.5235 \\
Restr. deviance & 294.9586 & Restr. log likelihood & -147.4793 \\
LR statistic & 14.43511 & Avg. log likelihood & -0.385334 \\
Prob(LR statistic) & 0.006028 & \multicolumn{5}{c}{364} \\
\hline ObswithDep=0 & 313 & Total obs \\
ObswithDep=1 & 51 & & \\
\hline
\end{tabular}




\section{Pensamiento Crítico Vol. ᄅl, № 2}

El cuadro 6 nos muestra que, al 5\% de error, solo los coeficientes estimados de las variables $\mathrm{X} 1$ y X2 son estadísticamente significativas. En tanto que los coeficientes de X3 y X4 no tienen significancia estadística.

El signo de la variable X1 es positivo, en tanto que el de la variable X2 es negativo.

El signo positivo de la variable X1 indica que la probabilidad de que el niño sea víctima de algún robo o asalto en la calle es mayor en la medida en que este viva solo con la madre o el padre.

El signo negativo de la variable $\mathrm{X} 2$ pone de manifiesto que la probabilidad de que el niño sufra algún robo o asalto en la calle es mayor en tanto que este confíe poco o nada en sus padres.

En resumen, la regresión econométrica indica que la probabilidad de que el niño sea asaltado en la calle es mayor en la medida en que este pertenezca a un hogar disfuncional (el niño no vive con la madre y el padre juntos) y que tenga poca o ninguna confianza en sus progenitores.

El test o la prueba de Hosmer - Lemeshownos permite efectuar comparaciones entre el valor estimado y el observado por grupos (que en nuestro caso es 10) y a su vez contrastar si las observaciones se asemejan a las estimaciones del modelo Logit. En el cuadro 7 se observa un Prob. Chi-Sq(8) $=0.0595>\alpha=0.05$, por la que podemos afirmar que la función de probabilidad del modelo tiene similitud a la distribución logística.

El cuadro 8 de la predicción de expectativas con punto de corte 0.5 muestra que de los 313 niños encuestados que mencionaron que no fueron asaltados, efectivamente todos no fueron asaltados, lo cual nos indica que la especificidad del modelo es del 100\%. En tanto que el modelo no predijo ningún caso de asalto, implica que la sensibilidad del modelo es 0 . Con lo cual se puede afirmar que este modelo solo permite pronosticar casos de no asalto. 


\section{Juan León Mendoza}

\section{CUADRO7 \\ Test de Hosmer-Lerneshow}

Goodness-of-Fit Evaluation for Binary Specification

Andrews and Hosmer-Lemeshow Tests

Equation: UNTITLED

Date: 12/06/15 Time: 10:34

Grouping based upon predicted risk (randomize ties)

\begin{tabular}{|c|c|c|c|c|c|c|c|c|}
\hline & \multicolumn{3}{|c|}{ Quantile of Risk } & \multicolumn{2}{|c|}{ Dep $=0$} & \multirow{2}{*}{$\begin{array}{l}\text { Dep=1 } \\
\text { Expect }\end{array}$} & \multirow{2}{*}{$\begin{array}{l}\text { Total } \\
\text { Obs }\end{array}$} & \multirow{2}{*}{$\begin{array}{c}\mathrm{H}-\mathrm{L} \\
\text { Value }\end{array}$} \\
\hline & Low & High & Actual & Expect & Actual & & & \\
\hline 1 & 0.0794 & 0.0875 & 32 & 33.0840 & 4 & 2.91602 & 36 & 0.43847 \\
\hline 2 & 0.0875 & 0.0875 & 36 & 32.8512 & 0 & 3.14882 & 36 & 3.45064 \\
\hline 3 & 0.0875 & 0.0875 & 32 & 33.7618 & 5 & 3.23824 & 37 & 1.05041 \\
\hline 4 & 0.0962 & 0.0963 & 33 & 32.5348 & 3 & 3.46519 & 36 & 0.06910 \\
\hline 5 & 0.0963 & 0.1057 & 31 & 33.3715 & 6 & 3.62851 & 37 & 1.71847 \\
\hline 6 & 0.1058 & 0.1306 & 29 & 31.8512 & 7 & 4.14878 & 36 & 2.21471 \\
\hline 7 & 0.1306 & 0.1430 & 33 & 31.1119 & 3 & 4.88809 & 36 & 0.84388 \\
\hline 8 & 0.1430 & 0.1947 & 32 & 30.9454 & 5 & 6.05461 & 37 & 0.21964 \\
\hline 9 & 0.1947 & 0.2488 & 33 & 28.2695 & 3 & 7.73053 & 36 & 3.68634 \\
\hline \multirow[t]{2}{*}{10} & 0.2490 & 0.4487 & 22 & 25.2188 & 15 & 11.7812 & 37 & 1.29026 \\
\hline & & Total & 313 & 313.000 & 51 & 51.0000 & 364 & 14.9819 \\
\hline \multicolumn{2}{|c|}{ H-L Statistic } & & 14.9819 & & \multicolumn{2}{|c|}{ Prob. Chi-Sq(8) } & 0.0595 & \\
\hline \multicolumn{2}{|c|}{ Andrews Statistic } & & 52.9986 & & \multicolumn{2}{|c|}{ Prob. Chi-Sq(10) } & 0.0000 & \\
\hline
\end{tabular}

\section{CUADRO 8}

Predicción de expectativas

Expectation-Prediction Evaluation for Binary Specification

Equation: UNTITLED

Date: 12/06/15 Time: 10:32

Successcutoff: $\mathrm{C}=0.5$

\begin{tabular}{lcccccc}
\hline & \multicolumn{3}{c}{ EstimatedEquation } & \multicolumn{3}{c}{ ConstantProbability } \\
& Dep $=0$ & Dep $=1$ & Total & Dep $=0$ & Dep $=1$ & Total \\
\hline $\mathrm{P}($ Dep $=1)<=$ C & 313 & 51 & 364 & 313 & 51 & 364 \\
$\mathrm{P}($ Dep $=1)>C$ & 0 & 0 & 0 & 0 & 0 & 0 \\
Total & 313 & 51 & 364 & 313 & 51 & 364 \\
Correct & 313 & 0 & 313 & 313 & 0 & 313 \\
\% Correct & 100.00 & 0.00 & 85.99 & 100.00 & 0.00 & 85.99
\end{tabular}


Pensamiento Crítico Vol. ᄅl, № 2

\begin{tabular}{|c|c|c|c|c|c|c|}
\hline \% Incorrect & 0.00 & 100.00 & 14.01 & 0.00 & 100.00 & 14.01 \\
\hline Total Gain* & 0.00 & 0.00 & 0.00 & & & \\
\hline \multirow[t]{3}{*}{ PercentGain** } & NA & 0.00 & 0.00 & & & \\
\hline & \multicolumn{3}{|c|}{ EstimatedEquation } & \multicolumn{3}{|c|}{ ConstantProbability } \\
\hline & Dep $=0$ & $\mathrm{Dep}=1$ & Total & $\mathrm{Dep}=0$ & Dep $=1$ & Total \\
\hline$E(\#$ of $D e p=0)$ & 271.31 & 41.69 & 313.00 & 269.15 & 43.85 & 313.00 \\
\hline$E(\#$ of $D e p=1)$ & 41.69 & 9.31 & 51.00 & 43.85 & 7.15 & 51.00 \\
\hline Total & 313.00 & 51.00 & 364.00 & 313.00 & 51.00 & 364.00 \\
\hline Correct & 271.31 & 9.31 & 280.62 & 269.15 & 7.15 & 276.29 \\
\hline \% Correct & 86.68 & 18.25 & 77.09 & 85.99 & 14.01 & 75.90 \\
\hline$\%$ Incorrect & 13.32 & 81.75 & 22.91 & 14.01 & 85.99 & 24.10 \\
\hline Total Gain* & 0.69 & 4.24 & 1.19 & & & \\
\hline PercentGain** & 4.93 & 4.93 & 4.93 & & & \\
\hline
\end{tabular}

*Change in "\% Correct" from default (constant probability) specification

**Percent of incorrect (default) prediction corrected by equation

\section{Discusión de resultados}

En principio, la mayoría absoluta de los niños de 8 a 12 años de edad encuestados manifestaron estar muy preocupados por el fenómeno de la violencia e inseguridad urbana reinante en Lima Metropolitana y Callao, lo cual de alguna manera estaría influyendo negativamente en su formación personal y emocional, posibilitando a su vez que en el futuro sean ciudadanos violentos.

Según la percepción de los niños, el robo y el asalto en la calle es el problema de violencia e inseguridad más importante, seguido por el consumo de drogas, el bullying, la violación y los asesinatos.

La percepción de los niños sobre los robos y los asaltos como el principal problema guarda concordancia con la información estadística reportada por el INEI (2015) sobre la percepción de la violencia de los adultos. En ella se señala que este tipo de delitos son los que predominan tanto en el Perú en general como también en la capital peruana. 


\section{Juan León Mendoza}

Un análisis comparativo entre el índice de victimización a nivel de los niños y de los adultos muestra que la tasa de robos y asaltos en la calle hacia los niños es aproximadamente la mitad de lo que ocurre a las personas adultas: el 14\% de los niños fueron víctimas de este tipo de delitos; a nivel de adultos la tasa es $28,8 \%$ (ver cuadro 9). Este hecho refleja que el problema de los robos y los asaltos en la calle afecta no solo a los adultos, sino también a los niños, aunque en menor grado.

\section{CUADRO 9}

Cuadro comparativo del índice de victimización

\begin{tabular}{|l|c|c|}
\hline Tipo de violencia & $\begin{array}{c}\text { Niños: Lima Metropolitana } \\
\text { y Callao (\%) }\end{array}$ & $\begin{array}{c}\text { Adultos: Lima } \\
\text { Metropolitana (\%) }\end{array}$ \\
\hline Sufrió robo en la calle & 14,0 & 28,8 \\
\hline Fue agredido u hostigado físicamente en la calle & 12,6 & 14,4 \\
\hline Su vivienda fue objeto de robo & 12,1 & 13,4 \\
\hline Ha sido víctima de acoso sexual callejero & 8,2 & 12,9 \\
\hline
\end{tabular}

Fuente: Encuesta ejecutada y Lima Cómo Vamos 2014.

La tasa de victimización de violencia física en las calles es aproximadamente similar tanto para los niños como para los adultos. Sin embargo, la tasa de violencia física contra los niños se produce en mayor magnitud en el hogar que en la calle. Este resultado indica que los hogares se están convirtiendo en principales focos de violencia física contra los niños y que coadyuvaría a que en el futuro siga reproduciéndose dicho fenómeno: los niños de ahora que crecen en un ambiente familiar de violencia física mañana tenderán a reproducir los mismos patrones de comportamiento con sus descendientes.

Contra lo que se podría esperar, la tasa de violencia física contra los niños no se produce en mayor magnitud en distritos u hogares de mayor pobreza. Más bien es mayor en sectores relativamente más pudientes (distritos donde predominan los sectores A y B). Pareciera que disponer de un mayor nivel de ingreso o estatus socioeconómico no garantiza necesariamente que los padres formen de manera adecuada o sin violencia a sus hijos menores.

A su vez, el hecho de que la tasa de violencia física contra los niños en el hogar sea mucho mayor $(42,0 \%)$ que contra las niñas $(28,3 \%)$ podría estar coadyuvando a la 


\section{Pensamiento Crítico Vol. ᄅl, № 2}

formación de generaciones masculinas con una cultura del machismo violento, es decir, una cultura en la que el hombre es más agresivo y de mayor fuerza bruta que la mujer.

La violencia sexual callejera en Lima Metropolitana y Callao no solo se produce a nivel de las personas adultas, sino también a nivel de los niños. Si bien la tasa de victimización de este tipo de violencia es mayor en la población adulta, la tasa a nivel de los niños no está muy lejos de ella.

Según la encuesta, la mayor parte de los niños se sienten muy seguros en su hogar y el colegio, en tanto que en las calles de su barrio u otros barrios, la mayor parte se siente poco segura o insegura. Entonces, el problema de la percepción de inseguridad de los niños se localiza básicamente en las calles. Sin embargo, la percepción de inseguridad de los niños en las calles de Lima y Callao es menor que la de los adultos.

Si bien la sensación de inseguridad en las calles es mayor entre las personas adultas que entre los niños, en términos de estratos socioeconómicos, existe una similitud de tendencia: tanto a nivel de los niños como a nivel de los adultos, la tasa de percepción de inseguridad es mayor en la medida en que disminuye el nivel socioeconómico.

La mayoría de los niños perciben que el problema de la violencia e inseguridad ha aumentado en los últimos doce meses. Pero, también en forma similar que los adultos, en su mayoría opinan que dicho problema seguirá igual o será más grave en los próximos doce meses (ver cuadro 10). El pesimismo sobre la solución del problema es general, tanto a nivel de los niños como en el caso de los adultos. Sin embargo, los que opinan que dicho problema será resuelto (aunque en un porcentaje bajo) es mayor en los niños que en los adultos: los niños son relativamente menos pesimistas.

CUADRO 10

Cuadro comparativo sobre las perspectivas de solución del problema de la violencia e inseguridad urbana para el próximo año

\begin{tabular}{|l|l|l|}
\hline Perspectivas & Niños: Lima Metropolitana y Callao (\%) & Adultos: Lima Metropolitana (\%) \\
\hline Será resuelto & 16,2 & 4 \\
\hline Seguirá igual & 50,3 & 51 \\
\hline Será más grave & 31,0 & 40 \\
\hline ns/nr & 2,5 & 6 \\
\hline
\end{tabular}

Fuente: Encuesta ejecutada y Pulso Perú 2015. 


\section{Juan León Mendoza}

Los niños confían básicamente en sus padres y sus profesores; pero, mayoritariamente, confían poco o no confían en la Policía, el serenazgo, sus compañeros de colegio y sus vecinos mayores. Es decir, no confían en aquellas instituciones encargadas de velar por la seguridad y el orden ciudadano. A su vez, se debe destacar el hecho de que las niñas muestran un menor grado de confianza que los niños en todas las instancias o agentes considerados (padres, profesores, compañeros de colegio, la Policía, el serenazgo, vecinos mayores de edad).

El hecho de que las niñas sientan un menor grado de confianza en todas las instituciones señaladas podría posibilitar, hasta cierto punto, que en el futuro se tenga una mayor cantidad de mujeres que varones con una relativa menor dotación del capital social, es decir, personas con menor capacidad y praxis de confianza, cooperación y sinergia.

La percepción de los niños sobre los factores que explican el hecho de que las personas se dediquen al delito del robo y el asalto difiere, de alguna manera, de los resultados hallados en algunos estudios efectuados sobre dicho fenómeno a nivel de las personas adultas.

Por ejemplo, León (2015), en una investigación econométrica sobre los determinantes de los robos y asaltos en Lima Metropolitana y Callao, encontró que entre los principales factores explicativos están la desigualdad en la distribución de ingresos, el consumo de droga, la densidad poblacional y la pobreza. En tanto que, según la percepción de los niños, los robos y los asaltos en las calles se deben básicamente al hecho de que los que cometen dicho delito son personas a las que no les gusta trabajar, les faltó una buena educación en casa o no tuvieron una buena niñez.

Los niños, en su mayoría, son partidarios de un castigo gradual a los delincuentes: un $45,6 \%$ sugiere que se les debe meter a la cárcel por un tiempo hasta que se reformen y un $35 \%$ plantea que deben ser sancionados con cárcel perpetua. Sin embargo, existe un porcentaje, aunque pequeño (10,4\% de los encuestados), que plantea un castigo más radical: aplicarles la pena de muerte.

Con la finalidad de reducir o resolver el problema de la delincuencia urbana, los niños sugieren que se incremente el número de policías. Sin embargo, no solo plantean que se eleve la dotación policial, sino que aumente su eficacia en la captura de los delincuentes. Solicitan mayor eficacia y cantidad de policías. También creen que se debe aplicar un 


\section{Pensamiento Crítico Vol. ᄅl, № 2}

castigo más fuerte a los que delinquen, y piden que se coloquen más cámaras de vigilancia. Estas opiniones indican que los niños perciben como relativamente suave el castigo a los delincuentes y que las dotaciones de las cámaras de vigilancia son insuficientes.

Finalmente, la regresión econométrica efectuada indica que la probabilidad de ser víctima de un asalto o robo en la calle es mayor en la medida en que el niño pertenezca a un hogar disfuncional y tenga poca o ninguna confianza en sus padres. Es decir, en tanto que el niño viva solo con la madre o el padre o algún familiar (no con el padre y la madre en conjunto), es más probable que sea víctima de la ola delincuencial en la calle. En el mismo sentido, la falta de confianza de los niños en sus padres eleva la probabilidad de ser víctima de este tipo de delitos.

Lo señalado pone de manifiesto que para minimizar la tasa de victimización y coadyuvar a un desarrollo adecuado de los niños, es de máxima importancia que se establezcan hogares sólidamente constituidos, y se cultive la cultura de la confianza dentro del seno familiar.

\section{Recomendaciones}

En aras de la formación de futuros ciudadanos no muy propensos a la violencia, se sugieren las siguientes acciones o líneas de política:

a) Las autoridades nacionales, locales y las instituciones en general deben priorizar la lucha contra el robo callejero, el consumo de las drogas y los actos de violación.

b) Se debe implementar, de manera institucionalizada y agresiva, la Escuela para padres. Ello no solo para minimizar la cantidad de hogares disfuncionales, sino también para ver la manera en que se puede elevar el grado de confianza de los niños en sus padres.

c) La Policía Nacional del Perú debería implementar actividades y programas que permitan que los niños eleven su confianza en la institución policial.

d) Se debe incrementar el número de policías y mejorar su eficiencia operativa. 


\section{Juan León Mendoza}

e) En las escuelas y colegios se deben ejecutar actividades para que los niños internalicen la cultura de la confianza en sus compañeros.

f) Las autoridades $e$ instituciones deben promover la cultura del esfuerzo, la responsabilidad y el trabajo honrado.

\section{Referencias bibliográficas}

Del Olmo, R. (2000). Ciudades duras y violencia urbana. Nueva Sociedad,(167),74 - 86. Disponible en: http://biblioteca2012.hegoa.efaber.net/system/ebooks/9204/ original/Inseguridad_Violencia_y_Miedo_en_AL.pdf

Dodge, K.; Bates, J. y Pettit, G. (1990). Mechanisms in the cycle of violence. Science, 250 (4988), 1678-1683. Disponible en: http://www.columbia.edu/cu/psychology/ courses/3615/Readings/Science_1990_Dodge_1678_83.pdf

Hofstetter, M. (1998). La violencia en los modelos de crecimiento económico. Revista de Economía de la Universidad del Rosario, 1(2), p. 67-77.Disponible en: http://revistas. urosario.edu.co/index.php/economia/article/view/978.

Instituto Nacional de Estadística e Informática (2015). Estadísticas de seguridad Ciudadana. Informe Técnico (3). Disponible en: https://www.inei.gob.pe/media/ MenuRecursivo/boletines/boletinseguruidad.pdf

León, J. (2015). Determinantes de la violencia urbana en Lima Metropolitana y Callao: Casos de delitos contra el patrimonio. Pensamiento Crítico, 20 (1), p. 75 - 96. Disponible en: http://revistasinvestigacion.unmsm.edu.pe/index.php/econo/article/ view/11485/10331

Municipalidad de Lima Metropolitana (2014).Encuesta Lima Cómo Vamos 2014. Disponible en: http://www.limacomovamos.org/

Oliveros, M.; Figueroa, L; Mayorga; Cano, B; Quispe, Y. y Barrientos, A. (2008). Violencia escolar (bullying) en colegios estatales de primaria en el Perú. Revista Peruana de Pediatría, 61 (4), p. 215 - 220.Disponible en: http://revistas.concytec. gob.pe/pdf/rpp/v61n4/a04v61n4.pdf 


\section{Pensamiento Crítico Vol. ᄅl, № 2}

Organización de las Naciones Unidas (2006). Las voces de niñas, niños y adolescentes sobre la violencia. Washington D. C. Disponible en: http://www.unicef.org/ecuador/ Estudio_violencia_contra.pdf

Organización Panamericana de la Salud (2002). Informe Mundial sobre la violencia y la salud. Washington D. C. Disponible en: http://www.who.int/violence_injury_ prevention/violence/world_report/en/summary_es.pdf

Osofsky, J. (1999). The impact of violence on children. Domestic Violence and Children, 9 (3),p. 33-49. Disponible en:https://www.princeton.edu/futureofchildren/publications/ docs/09_03_2.pdf

Perry, B. (1997). Incubated in terror: neorodevelopmental factors in the cycle violence. En Osofsky (ed.). Children, Yoth, and Violence: The search for solutions. New York, : Gilford Press. Disponible en: https://childtrauma.org/wwwtent/uploads/2013/11/ Incubated_In_Terror.pdf

Ponce, S. (1995). Estudio epidemiológico sobre el maltrato infantil en la población escolarizada de Lima Metropolitana y Callao. CEDRO, Lima.

Sharkey, P. (2010).The acute effect of local homicides on children's cognitive performance. Current Issue, 07 (26), p. 11733-11738. Disponible en: http://www. pnas.org/content/107/26/11733.full

Sheidow, A., Gorman-Smith, D., Tolan, P. y Henry, D. (2001).Family and community characteristics: Risk factors violence exposure in inner-city youth. Journal of Community Psychology, 29 (3), p. 345-360. Disponible en: http://onlinelibrary.wiley. com/doi/10.1002/jcop.1021/abstract

Vanderschueren, F. (1994). La violencia urbana, los pobres de la ciudad y la justicia. En: Concha Eastman, A., Carrión, F., Cobo, G. (eds.). Ciudad y violencia en América Latina. Programa de Gestión Urbana, Quito. 